\title{
Strong terahertz radiation generation by beating of two x- mode spatial triangular lasers in magnetized plasma
}

\author{
PRATEEK VARSHNEY, ${ }^{1}$ VIVEK SAJAL, ${ }^{1}$ SWETA BALIYAN, ${ }^{2}$ NAVNEET K. SHARMA, ${ }^{1}$ \\ PRASHANT K. CHAUHAN, ${ }^{1}$ AND RAVINDRA KUMAR ${ }^{1}$ \\ ${ }^{1}$ Department of Physics and Materials Science and Engineering, Jaypee Institute of Information Technology, Uttar Pradesh, India \\ ${ }^{2}$ Department of Applied Sciences and Humanities, ABES Engineering College Ghaziabad, Ghaziabad, India
}

(RECEIVED 4 August 2014; ACCEPTED 16 September 2014)

\begin{abstract}
Resonant THz radiation generation is proposed by beating of two spatial-triangular laser pulses of different frequencies $\left(\omega_{1}, \omega_{2}\right)$ and wave numbers $\left(\vec{k}_{1}, \vec{k}_{2}\right)$ in plasma having external static magnetic field. Laser pulses co-propagating perpendicular to a dc magnetic field exert a nonlinear ponderomotive force on plasma electrons, imparting them an oscillatory velocity with finite transverse and longitudinal components. Oscillatory plasma electrons couple with periodic density ripples $n^{\prime}=n_{q 0} e^{i q z}$ to produce a nonlinear current, i.e., responsible for resonantly driving terahertz radiation at $\left(\omega=\omega_{1}-\omega_{2}, \vec{k}=\vec{k}_{1}-\vec{k}_{2}+\vec{q}\right)$. Effects of $\mathrm{THz}$ wave frequency, laser beam width, density ripples, and applied magnetic field are studied for the efficient $\mathrm{THz}$ radiation generation. The frequency and amplitude of $\mathrm{THz}$ radiation were observed to be better tuned by varying dc magnetic field strength and parameters of density ripples (amplitude and periodicity). An efficiency about 0.02 is achieved for laser intensity of $2 \times 10^{15} \mathrm{~W} / \mathrm{cm}^{2}$ in a plasma having density ripples about 30\%, plasma frequency about $1 \mathrm{THz}$ and magnetic field about $100 \mathrm{kG}$.
\end{abstract}

Keywords: Resonant Terahertz generation; Rippled magnetized plasma; Triangular laser

\section{INTRODUCTION}

Compact powerful $\mathrm{THz}$ sources $(0.1-10 \mathrm{THz})$ with fields as high as a few $\mathrm{MV} / \mathrm{cm}$ (or power about $\mathrm{GW} / \mathrm{cm}^{2}$ ) are desirable for scientific and commercial applications such as rapid twodimensional imaging (Dragoman \& Dragoman, 2004), topography (Siegel, 2002), remote sensing (Sizov, 2010), chemical and security identification (Leemans et al., 2003), outer space communication and submillimeter radars (Schroeder et al., 2004), imaging of biological tissue (Carr et al., 2002), spectroscopic identifications of complex molecules (Abo-Bakar et al., 2003), explosive detection (Yoshii et al., 1997), etc. It is difficult to obtain such powerful THz emitters utilizing electrooptic crystals (Hamester et al., 1993; 1994), semiconductors (Schillinger \& Sauerbrey, 1999), photoconductive antennas (Sprangle et al., 2004), etc., due to the breakdown limit and low conversion efficiencies. To address these issues, plasma based THz emitters utilizing electron beams and laser plasma interaction were proposed such as coherent radiation from

Address correspondence and reprint requests to: Vivek Sajal, Department of Physics and Materials Science and Engineering, Jaypee Institute of Information Technology, Noida-201307, Uttar Pradesh, India. E-mail: vsajal@rediffmail.com plasma oscillations driven by ultra-short laser pulses (Antonesen et al., 2006), synchrotron radiation from accelerated electrons (Liu et al., 2009), and transition radiation of electron beams (Sheng et al., 2004) etc. In these schemes, plasma as a nonlinear medium can handle very high power lasers with an added advantage of not having damage limit (Sheng et al., 2005; Kumar \& Tripathi, 2013; Verma \& Sharma, 2009; Giulietti et al., 1988; Panwar et al., 2013; Ghorbanalilu, 2012; Garg \& Tripathi, 2010; Verma \& Sharma, 2011).

Recently, various experiments based on laser beating (one of fundamental and other at second harmonics) in plasmas have reported efficient $\mathrm{THz}$ radiation generation (Esarey et al., 1996; D'Amico et al., 2007; Tani et al., 2000; Xie et al., 2006; Bhasin \& Tripathi, 2009; Tripathi \& Liu, 1990; Liu \& Tripathi, 2009; Dua et al., 2011; Hu et al., 2010; Varshney et al., 2014). Out of various schemes based on laser plasma interaction, $\mathrm{THz}$ radiation generation by beating of two lasers of different frequencies and wave numbers in plasmas has shown tremendous potential in terms of amplitude, tunability, efficiency, and directionality (Varshney et al., 2013; 2014; Nafil et al., 2013; Malik et al., 2012; 2011; Malik \& Malik, 2012). THz sources 
based on beating can also be scaled to high peak powers. Xie et al. (2006) observed that properties of emitted $\mathrm{THz}$ radiation are consistent with four waves mixing in plasma, and $\mathrm{THz}$ emission is maximized when the polarization of the laser beams and the $\mathrm{THz}$ are aligned. Bhasin and Tripathi (2011) and Varshney et al. (2013) have examined resonant $\mathrm{THz}$ radiation generation by beating of $x$-polarized laser beams in rippled density magnetized plasma.

Different types of laser profiles (Nafil et al., 2013, Malik et al., 2012; 2011) were utilized by various researchers to enhance amplitude and power of $\mathrm{THz}$ radiation in various beat wave schemes in plasmas. Malik et al. (2012) utilized the Gaussian profile of beating lasers in periodic density rippled plasma and realized efficiency and $10^{-3}$ for the laser intensity of about $10^{14} \mathrm{~W} / \mathrm{cm}^{2}$. The efficiency is improved to about 0.006 by replacing the Gaussian profile by super Gaussian profile (Malik \& Malik, 2012) of beating lasers for same parameters. They concluded that the two spatial super Gaussian lasers of higher index and smaller beam width can produce much stronger $\mathrm{THz}$ radiation compared to the case of Gaussian lasers. The radiation can also be focused at a desired position along with more collimation by varying of the index of lasers in plasma having larger density ripples. Malik et al. (2012) have achieved efficiency about $10^{-2}$ by beating of spatial triangular lasers in plasma for the laser intensity about $10^{14} \mathrm{~W} / \mathrm{Cm}^{2}$, at the $\mathrm{THz}$ field about $10^{5} \mathrm{kV} / \mathrm{cm}$.

In the present work, we propose a scheme of resonant $\mathrm{THz}$ radiation generation by beating of two extraordinary lasers (with triangular envelopes in space) co-propagating in a plasma having periodically modulated density ripples. The issue of high amplitude and power of $\mathrm{THz}$ radiation is addressed by employing triangular profiles and $\mathrm{X}$-mode polarization of lasers. Both the beating lasers and generated $\mathrm{THz}$ radiation have the same state of polarization because the $\mathrm{THz}$ emission is maximized when the polarization of laser beams and the THz are aligned (Malik et al., 2012). Issues of directionality and tunability are addressed by applying dc static magnetic field in transverse direction. Two co-propagating $\mathrm{x}$-mode triangular shaped $\left[\left(\omega_{1}, \vec{k}_{1}\right)\right.$ and $\left.\left(\omega_{2}, \overrightarrow{k_{2}}\right)\right]$ (Fig. 1) exert a nonlinear ponderomotive force at frequency $\omega=\omega_{1}-\omega_{2}$ and wave number $\vec{k}=\overrightarrow{k_{1}}-\overrightarrow{k_{2}}$ on plasma electrons. Velocity perturbation due to ponderomotive forces couples with density ripples of appropriate periodicity and excites a nonlinear current. The nonlinear current can excite $\mathrm{THz}$ radiation if resonance conditions are simultaneously satisfied. In Section 2, expressions of ponderomotive force, density perturbation and nonlinear current density are derived. The amplitude and efficiency of $\mathrm{THz}$ wave are calculated in Section 3. Discussion on results is given in the Section 4.

\section{NONLINEAR CURRENT DUE TO BEATING OF LASERS}

Consider a laser produced rippled plasma of density $n \equiv n_{0}+$ $n^{\prime}, n^{\prime}=n_{q 0} e^{i q z}$ with static magnetic field $\vec{B}_{0}$ in $\hat{x}$ direction, where $n_{q 0}$ as the amplitude of ripple and $q$ as the wave vector of density ripples. Plasma density ripples can be produced using various techniques involving transmission ring grating and patterned mask, where the control of ripple parameters might be possible by changing groove period, groove structure, and duty cycle in such a grating, and by adjusting the period and the size of mask (Malik \& Malik, 2012; Penano et al., 2010). Two x-mode spatial triangular lasers co-propagate through it along $\hat{z}$-direction having electric field profile (Malik et al., 2012):

$$
\vec{E}_{j}=\left(\hat{y}-\frac{\varepsilon_{j z y}}{\varepsilon_{j z z}} \hat{z}\right) E_{00} e^{-i\left(\omega_{j} t-k_{j} z\right)}
$$

where $E_{00}^{2}=A_{0}^{2}\left(1-\left|y / a_{0}\right|\right)^{2}$, for $\left|y / a_{0}\right|<1$ and zero otherwise; $j=1,2 ; \quad k_{j}=\frac{\omega_{j}}{c}\left(1-\frac{\omega_{p}^{2}}{\omega_{j}^{2}} \frac{\omega_{j}^{2}-\omega_{p}^{2}}{\omega_{j}^{2}-\omega_{h}^{2}}\right)^{1 / 2} ; a_{0}$ is the beam width parameter of lasers, $\omega_{h}^{2}=\omega_{p}^{2}+\omega_{c}^{2}, \quad \omega_{p}^{2}=$ $4 \pi n_{0} e^{2} / m$ and $\omega_{c}=e B_{0} / m$ are upper hybrid frequency, electron plasma frequency, and cyclotron frequency, respectively; $-e$ and $m$ are the electronic charge and mass; and $\varepsilon_{j z z}=\left[1-\omega_{p}^{2} /\left(\omega_{j}^{2}-\omega_{c}^{2}\right)\right] \quad \& \quad \varepsilon_{j z y}=-i\left[\omega_{c} \omega_{p}^{2} / \omega_{j}\left(\omega_{j}^{2}-\omega_{c}^{2}\right)\right]$ are components of the dielectric tensor $\varepsilon_{j}$. Extraordinary mode or X-mode is the natural electromagnetic mode of magnetized plasma.

If the electric field of propagating laser $\vec{E}_{j}=\hat{y} E_{00} e^{-i\left(\omega_{j} t-k_{j} z\right)}$ in a plasma is perpendicular to applied dc magnetic field

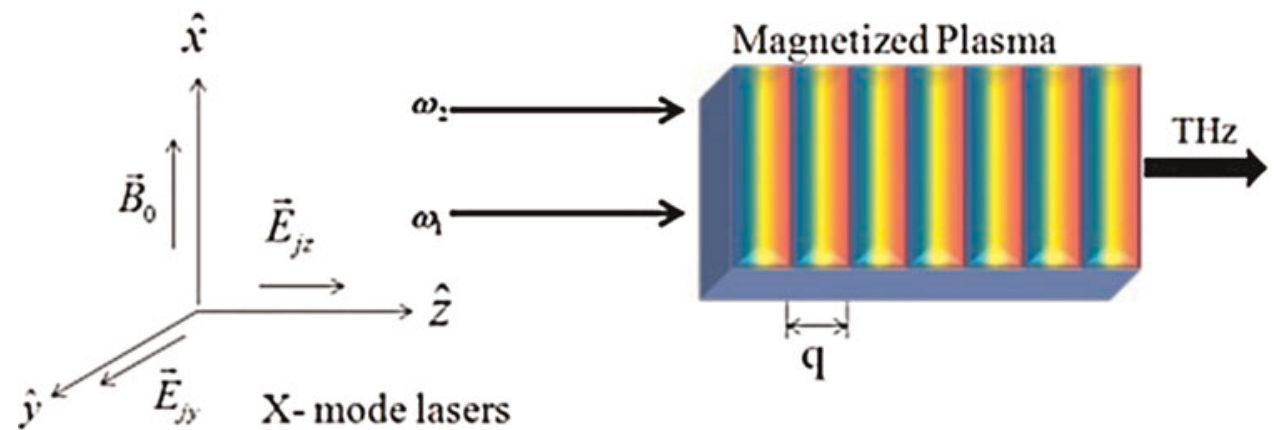

Fig. 1. Schematic diagram of terahertz radiation generation by beating of two $\mathrm{x}$-mode lasers. 
$\vec{B}_{0}=B_{0} \hat{x}$, then the plasma electron motion will also be affected by $-e\left(\vec{v} \times \vec{B}_{o}\right)$ force due to applied magnetic field and the dispersion relation will be changed. As a result, a longitudinal component develops and laser becomes partly longitudinal and partly transverse. These two components are related to each other by the expression $E_{z}=-\left(\varepsilon_{j z y} / \varepsilon_{j z z}\right) E_{y}$. This expression has the signature of applied dc magnetic field (Chen, 1983). These extraordinary modes of electromagnetic waves are extensively utilized in magnetized plasma for various purposes like parametric instabilities, electron acceleration, harmonic generation, etc. Here, lasers impart oscillatory velocity to plasma electrons in $\hat{y}$ and $\hat{z}$ direction, given by

$$
\begin{aligned}
& v_{j y}=\frac{e}{m\left(\omega_{j}^{2}-\omega_{c}^{2}\right)}\left[i \omega_{j}+\omega_{c} \frac{\varepsilon_{j z y}}{\varepsilon_{j z z}}\right] E_{00} e^{-i\left(\omega_{j} t-k_{j} z\right)}, \\
& v_{j z}=\frac{e}{m\left(\omega_{j}^{2}-\omega_{c}^{2}\right)}\left[\omega_{c}+i \omega_{j} \frac{\varepsilon_{j z y}}{\varepsilon_{j z z}}\right] E_{00} e^{-i\left(\omega_{j} t-k_{j} z\right)} .
\end{aligned}
$$

Lasers beat together and exert a ponderomotive force $\vec{F}_{p}\left(=F_{p y} \hat{y}+F_{p z} \hat{z}\right)$ on plasma electron at frequency $\omega=$ $\omega_{1}-\omega_{2}$ and wave vector $\vec{k}=\vec{k}_{1}-\vec{k}_{2}$. The components of the ponderomotive force $F_{p y}$ and $F_{p z}$ are as follows:

$$
\begin{aligned}
F_{p y}= & \frac{e^{2}}{2 m}\left[\frac { 1 } { ( \omega _ { 1 } ^ { 2 } - \omega _ { c } ^ { 2 } ) ( \omega _ { 2 } ^ { 2 } - \omega _ { c } ^ { 2 } ) } \left\{\frac{2}{a_{0}\left(1-\left|y / a_{0}\right|\right)}\right.\right. \\
& \times\left(i \omega_{1}+\omega_{c} \frac{\varepsilon_{1 z y}}{\varepsilon_{1 z z}}\right)\left(-i \omega_{2}+\omega_{c} \frac{\varepsilon_{2 z y}}{\varepsilon_{2 z z}}\right)+i k_{2}\left(\omega_{c}+i \omega_{1} \frac{\varepsilon_{1 z y}}{\varepsilon_{1 z z}}\right) \\
& \left.\times\left(-i \omega_{2}+\omega_{c} \frac{\varepsilon_{2 z y}}{\varepsilon_{2 z z}}\right)-i k_{1}\left(i \omega_{1}+\omega_{c} \frac{\varepsilon_{1 z y}}{\varepsilon_{1 z z}}\right)+\left(\omega_{c}-i \omega_{2} \frac{\varepsilon_{2 z y}}{\varepsilon_{2 z z}}\right)\right\} \\
& \left.+\frac{k_{2}\left(\omega_{c}+i \frac{\varepsilon_{1 z y}}{\varepsilon_{1 z z}} \omega_{1}\right)}{\omega_{2}\left(\omega_{1}^{2}-\omega_{c}^{2}\right)}+\frac{k_{1}\left(\omega_{c}-i \frac{\varepsilon_{2 z y}}{\varepsilon_{2 z z}} \omega_{2}\right)}{\omega_{1}\left(\omega_{2}^{2}-\omega_{c}^{2}\right)}\right] E_{00}^{2} e^{-i(\omega t-k z),}
\end{aligned}
$$

$$
\begin{aligned}
F_{p z}= & \frac{e^{2}}{2 m}\left[\frac { 1 } { ( \omega _ { 1 } ^ { 2 } - \omega _ { c } ^ { 2 } ) ( \omega _ { 2 } ^ { 2 } - \omega _ { c } ^ { 2 } ) } \left\{\frac{1}{a_{0}\left(1-\left|y / a_{0}\right|\right)}\left(i \omega_{1}+\omega_{c} \frac{\varepsilon_{1 z y}}{\varepsilon_{1 z z}}\right)\right.\right. \\
& \times\left(\omega_{c}-i \omega_{2} \frac{\varepsilon_{2 z y}}{\varepsilon_{2 z z}}\right)+\frac{1}{a_{0}\left(1-\left|y / a_{0}\right|\right)}\left(-i \omega_{2}+\omega_{c} \frac{\varepsilon_{2 z y}}{\varepsilon_{2 z z}}\right) \\
& \left.\times\left(\omega_{c}+i \omega_{1} \frac{\varepsilon_{1 z y}}{\varepsilon_{1 z z}}\right)-i k\left(\omega_{c}+i \omega_{1} \frac{\varepsilon_{1 z y}}{\varepsilon_{1 z z}}\right)\left(\omega_{c}-i \omega_{2} \frac{\varepsilon_{2 z y}}{\varepsilon_{2 z z}}\right)\right\} \\
& \left.+\frac{k_{2}\left(i \omega_{1}+\omega_{c} \frac{\varepsilon_{1 z y}}{\varepsilon_{1 z z}}\right)}{\omega_{2}\left(\omega_{1}^{2}-\omega_{c}^{2}\right)}+\frac{k_{1}\left(-i \omega_{2}+\omega_{c} \frac{\varepsilon_{2 z y}}{\varepsilon_{2 z z}}\right)}{\omega_{1}\left(\omega_{2}^{2}-\omega_{c}^{2}\right)}\right] E_{00}^{2} e^{-i(\omega t-k z)} .
\end{aligned}
$$

The ponderomotive force drives space charge oscillation at $\omega=\omega_{1}-\omega_{2}$ and wave number $\overrightarrow{k_{1}}-\overrightarrow{k_{2}}$. Assuming the potential of space charge mode to be $\phi$, the oscillatory velocity of electron due to space charge mode along with ponderomotive force in the presence of static magnetic field can be expressed as follows:

$$
\begin{gathered}
v_{y}=\frac{1}{m\left(\omega^{2}-\omega_{c}^{2}\right)}\left[i e \omega \nabla \phi-\omega_{c} F_{p y}+i \omega F_{p z}\right] . \\
v_{z}=\frac{1}{m\left(\omega^{2}-\omega_{c}^{2}\right)}\left[e \omega_{c}(\nabla \phi)+\omega_{c} F_{p z}+i \omega F_{p y}\right] .
\end{gathered}
$$

The nonlinear velocity given by Eq. (5) along with continuity equation provide density perturbation $n=n^{L}+n^{N L}$, where

$$
\begin{gathered}
n^{L}=\frac{1}{4 \pi e} k^{2} \chi \phi, \\
n^{N L}=\frac{n_{0} k}{m \omega\left(\omega^{2}-\omega_{\mathrm{c}}^{2}\right)}\left[i \omega F_{p z}-\omega_{c} F_{p y}\right],
\end{gathered}
$$

and $\chi=-\omega_{p}^{2} /\left(\omega^{2}-\omega_{c}^{2}\right)$. Linear density perturbation $\left(n^{L}\right)$ is induced self-consistently by space charge field and nonlinear density perturbation $\left(n^{N L}\right)$ is the consequence of ponderomotive force. Here, density perturbation is assumed to be small as compared to the density ripple. Substituting $n=n^{L}+n^{N L}$ in the Poisson's equation $\nabla^{2} \phi=4 \pi n e$, we obtain

$$
\varepsilon \phi=-\frac{4 \pi e}{k^{2}} n^{N L},
$$

where, $\varepsilon=1+\chi$. Combining Eqs. (6)-(8), we have,

$$
\phi=-\frac{4 \pi n_{0} e}{m k \omega\left(\omega^{2}-\omega_{h}^{2}\right)}\left[i \omega F_{p z}-\omega_{c} F_{p y}\right] .
$$

Substituting this value of $\phi$ in Eq. (5), we obtain the oscillatory velocity components of plasma electrons:

$$
\begin{gathered}
v_{y}=\frac{1}{m\left(\omega^{2}-\omega_{h}^{2}\right)}\left[-\omega_{c} F_{p y}+i \omega F_{p z}\right], \\
v_{z}=\frac{\omega_{c}}{m\left(\omega^{2}-\omega_{h}^{2}\right)} F_{p z}+i \frac{\left(\omega^{2}-\omega_{p}^{2}\right)}{m\left(\omega^{2}-\omega_{h}^{2}\right) \omega} F_{p y} .
\end{gathered}
$$

Oscillations at $\left(\omega, \vec{k}_{1}-\vec{k}_{2}\right)$ in the presence of density ripple $n_{q o} e^{i q z}$ excite nonlinear current at $\left(\omega, \overrightarrow{k_{1}}-\overrightarrow{k_{2}}+\vec{q}\right)$ which can be written as

$$
\vec{J}^{N L}=-\frac{1}{2} n_{q 0} e \vec{v}_{\omega} e^{i q z}
$$

This oscillatory current is the source for the emission of $\mathrm{THz}$ radiation at the beating frequency $\omega$ which is the same as that of the ponderomotive force but its wave number is different. For strong $\mathrm{THz}$ radiation, plasma density ripples should be periodic, otherwise $\vec{k}\left(=\vec{k}_{1}-\vec{k}_{2}+\vec{q}\right)$ will exhibit nonperiodic behavior; resonance condition can't be achieved and maximum energy transfer will not take place and consequently a weak field $\mathrm{THz}$ radiation will be generated. 


\section{THZ RADIATION AMPLITUDE}

The following wave equation is solved to find the amplitude of the $\mathrm{THz}$ wave

$$
-\nabla^{2} \vec{E}+\nabla \cdot(\nabla \cdot \vec{E})=\frac{4 \pi i \omega}{c^{2}} \vec{J}+\frac{\omega^{2}}{c^{2}}(\varepsilon \cdot \vec{E}),
$$

where, $\varepsilon$ is the plasma permittivity tensor at $\omega$. Taking fast phase variations in the electric field profile of $\mathrm{THz}$ radiation as $\vec{E}=\vec{A}(z) e^{-i(\omega t-k z)}$, the wave equation (Eq. (12)) governing the propagation of $\mathrm{THz}$ waves can be split into $\hat{y}$ and $\hat{z}$ components as follows:

$$
\begin{gathered}
A_{z}=-\frac{4 \pi i}{\omega \varepsilon_{z z}} J_{z}^{N L}-\frac{\varepsilon_{z y}}{\varepsilon_{z z}} A_{y}, \\
k^{2} A_{y}-2 i k \frac{\partial A_{y}}{\partial z}-\frac{\partial^{2} A_{z}}{\partial z^{2}}=+\frac{4 \pi i \omega}{c^{2}} J_{y}^{N L}+\frac{\omega^{2}}{c^{2}}\left(\varepsilon_{y y} A_{y}+\varepsilon_{y z} A_{z}\right) .
\end{gathered}
$$

The excited $\mathrm{THz}$ mode will have same polarization state as the beating lasers. It will have both $y$ - and $z$-components of electric field corresponding to its $\mathrm{x}$-mode nature. These components will be related to Eq. (12a). Xie et al. (2006) also observed that $\mathrm{THz}$ emission will be maximized when the polarization of laser beams and the $\mathrm{THz}$ are aligned. By rearranging Eq. (12a) and Eq. (12b), we obtained the equation governing transverse component $A_{y}$ of $\mathrm{THz}$ radiation. Longitudinal component of $\mathrm{THz}$ can be calculated by Eq. (12b)

$$
\begin{gathered}
-2 i k \frac{\partial A_{y}}{\partial z}+\left(k^{2}-\frac{\omega^{2}}{c^{2}} \varepsilon_{y y}+\frac{\omega^{2}}{c^{2}} \frac{\varepsilon_{y z} \varepsilon_{z y}}{\varepsilon_{z z}}\right) A_{y} \\
=+\frac{4 \pi i \omega}{c^{2}} J_{y}^{N L}-\frac{\varepsilon_{y z}}{\varepsilon_{z z}} \frac{4 \pi i \omega}{c^{2}} J_{z}^{N L} .
\end{gathered}
$$

From Eq. (13), one can observe that resonant $\mathrm{THz}$ radiation generation demands to satisfy the following dispersion relation for exact phase matching condition in rippled magnetized plasma which provides the periodicity of rippled structure and suggests that the maximum energy transfer from beating lasers to $\mathrm{THz}$ radiation will take place at resonance condition:

$$
k^{2}-\frac{\omega^{2}}{c^{2}} \varepsilon_{y y}+\frac{\omega^{2}}{c^{2}} \frac{\varepsilon_{y z} \varepsilon_{z y}}{\varepsilon_{z z}}=0 .
$$

Substituting the phase matching condition in Eq. (13), we obtain the amplitude of $\mathrm{THz}$ radiation as follows:

$$
\begin{aligned}
\left|A_{y}\right|= & \frac{1}{4 k} \frac{n_{q 0}}{n_{0}} \frac{\omega \omega_{p}^{2}}{\omega^{2}-\omega_{h}^{2}} \frac{z}{e c^{2}}\left[i\left(\frac{\omega^{2}-\omega_{p}^{2}}{\omega}-\left|\frac{\varepsilon_{z y}}{\varepsilon_{z z}}\right| \omega_{c}\right) F_{p y}\right. \\
& \left.+\left(\omega_{c}-\omega\left|\frac{\varepsilon_{z y}}{\varepsilon_{z z}}\right|\right) F_{p z}\right] .
\end{aligned}
$$

The normalized amplitude of $\mathrm{THz}$ radiation can be written as follows:

$$
\begin{aligned}
\left|\frac{A_{y}}{A_{0}}\right|= & \frac{1}{4 k} \frac{n_{q 0}}{n_{0}} \frac{1}{\omega\left(\omega^{2}-\omega_{h}^{2}\right)} z\left[\left(\frac{\omega^{2}-1}{\omega}-\left|\frac{\varepsilon_{z y}}{\varepsilon_{z z}}\right| \omega_{c}\right) f_{p y}\right. \\
& \left.+\left(\omega_{c}-\omega\left|\frac{\varepsilon_{z y}}{\varepsilon_{z z}}\right|\right) f_{p z}\right],
\end{aligned}
$$

where $f_{p z}=i F_{p z}$ and $f_{p y}=i F_{p y}$. Eq. (16) reveals that the normalized $\mathrm{THz}$ amplitude is directly proportional to the normalized amplitude of density ripples $n_{q 0} / n_{0}$, thus $\mathrm{THz}$ field increases on increasing ripple amplitude. Its explanation lies in Eq. (11); higher the ripple amplitude, greater the number of electrons involving in the generation of oscillatory nonlinear current. Higher number of charge carriers results into higher nonlinear current $\left(\vec{J}^{N L}\right)$ leading to more efficient $\mathrm{THz}$ radiation.

The phase matching condition $q=(\omega / c) \mid \varepsilon_{y y}+\left(\varepsilon_{x y} \varepsilon_{y x} /\right.$ $\left.\varepsilon_{x x}\right)^{1 / 2}-1$ | for resonant excitation of $\mathrm{THz}$ radiation provides the estimate of periodicity of rippled structure for maximum energy transfer in this process. In Figure 2, normalized periodicity factor $\left(c q / \omega_{p}\right)$ of density rippled structure is plotted as a function of normalized $\mathrm{THz}$ frequency $\left(\omega / \omega_{p}\right)$ and normalized cyclotron frequency $\left(\omega_{c} / \omega_{p}\right)$. Periodicity factor $(q)$ decreases with $\mathrm{THz}$ wave frequency, increases with applied magnetic field and achieves maximum value as THz frequency $(\omega)$ approaches toward resonance value $\omega \sim \omega_{h}$. The wavelength $(\lambda=2 \pi / q)$ is inversely proportional to $q$, thus one can conclude that steep ripples at closer distances should be constructed for resonant excitation of $\mathrm{THz}$ radiation. The reason behind the requirement of smaller periodicity factor $q$ (or larger ripple wavelength $\lambda$ ) for the excitation of high frequency $\mathrm{THz}$ waves can be traced in resonance condition $\vec{k}=\vec{k}_{1}-\vec{k}_{2}+q$ and $\omega=\omega_{1}-\omega_{2}$. To increase $\omega$, one has to increase $\omega_{1}$ by keeping $\omega_{2}$ fixed i.e., $\Delta \omega=\Delta \omega_{1}$. Wave vectors $\vec{k}$ and $\vec{k}_{1}$ change corresponding to changes in $\omega$ and $\omega_{1}$ respectively [keeping $\overrightarrow{k_{2}}$ fixed]. Thus, $\Delta \vec{k}=\Delta \overrightarrow{k_{1}}+\Delta q$. We know that for $\mathrm{x}$-mode electromagnetic wave we have
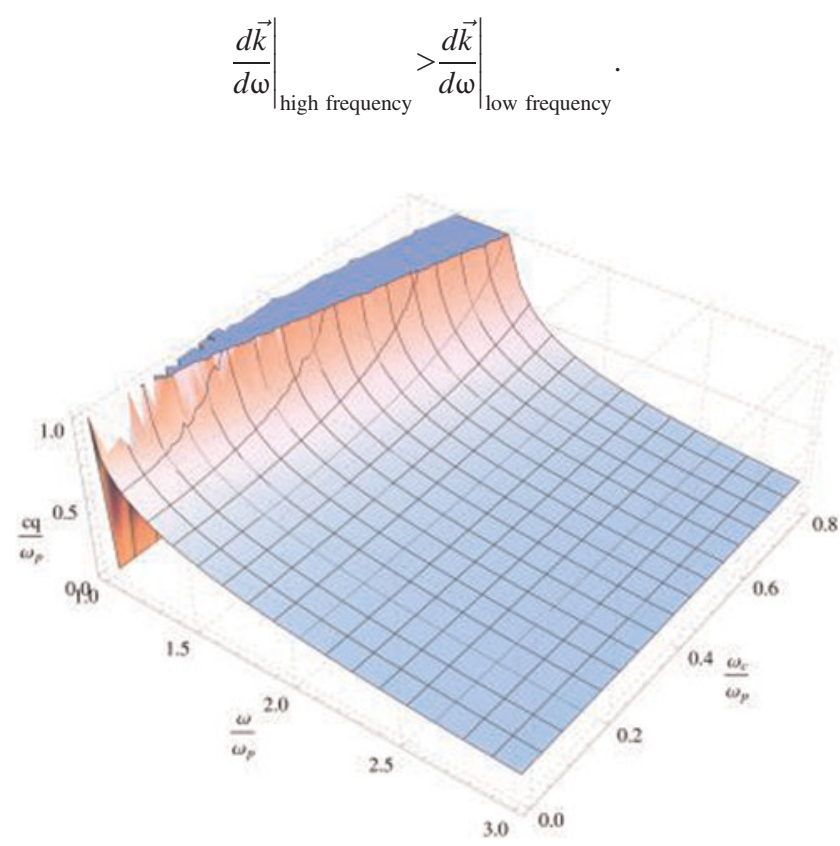

Fig. 2. Plot of the normalized ripple factor $q c / \omega_{p}$ as a function of the normalized $\mathrm{THz}$ wave frequency $\omega / \omega_{p}$ and normalized cyclotron frequency $\omega_{c} / \omega_{p}$. 
Thus, for equal change in frequency, change in wave vector for high frequency $\mathrm{x}$-wave will be greater than change in wave vector for low frequency $\mathrm{x}$-mode wave. Since, beating laser $\left(\omega_{1}, \vec{k}_{1}\right)$ and excited THz radiation $(\omega, \vec{k})$ have same state of polarization (x-mode polarization). In the present scheme, thus for equal change in frequency $\Delta \omega=\Delta \omega_{1}$ we have $\Delta \vec{k}<\Delta \overrightarrow{k_{1}}$. So, we can conclude that periodicity factor $q$ should be reduced (corresponding to $\Delta \vec{k}=\Delta \overrightarrow{k_{1}}+\Delta \vec{q}$ ) to enhance the frequency of excited $\mathrm{THz}$ radiation.

Figure 3a exhibits the variation of normalized $\mathrm{THz}$ wave amplitude $\left(A_{y} / 0.15 A\right)$ as a function of $\mathrm{THz}$ frequency $(v)$ and applied magnetic field $B_{c}$. Two mountain ranges of high $\mathrm{THz}$ amplitude excitation are observed which are corresponding to two propagating frequency regimes of extra ordinary electromagnetic wave in plasma. These two frequency regime are given by (1) $\omega_{L}<\omega<\omega_{h}$ (Region II of Fig. 3b) and (2) $\omega>\omega_{R}$ (Region IV of Fig. 3b), where $\omega_{L}=1 / 2\left[-\omega_{c}+\sqrt{\omega_{c}^{2}+4 \omega_{p}^{2}}\right]$ and $\omega_{R}=$ $1 / 2\left[\omega_{c}+\sqrt{\omega_{c}^{2}+4 \omega_{p}^{2}}\right]$. These two mountains are separated
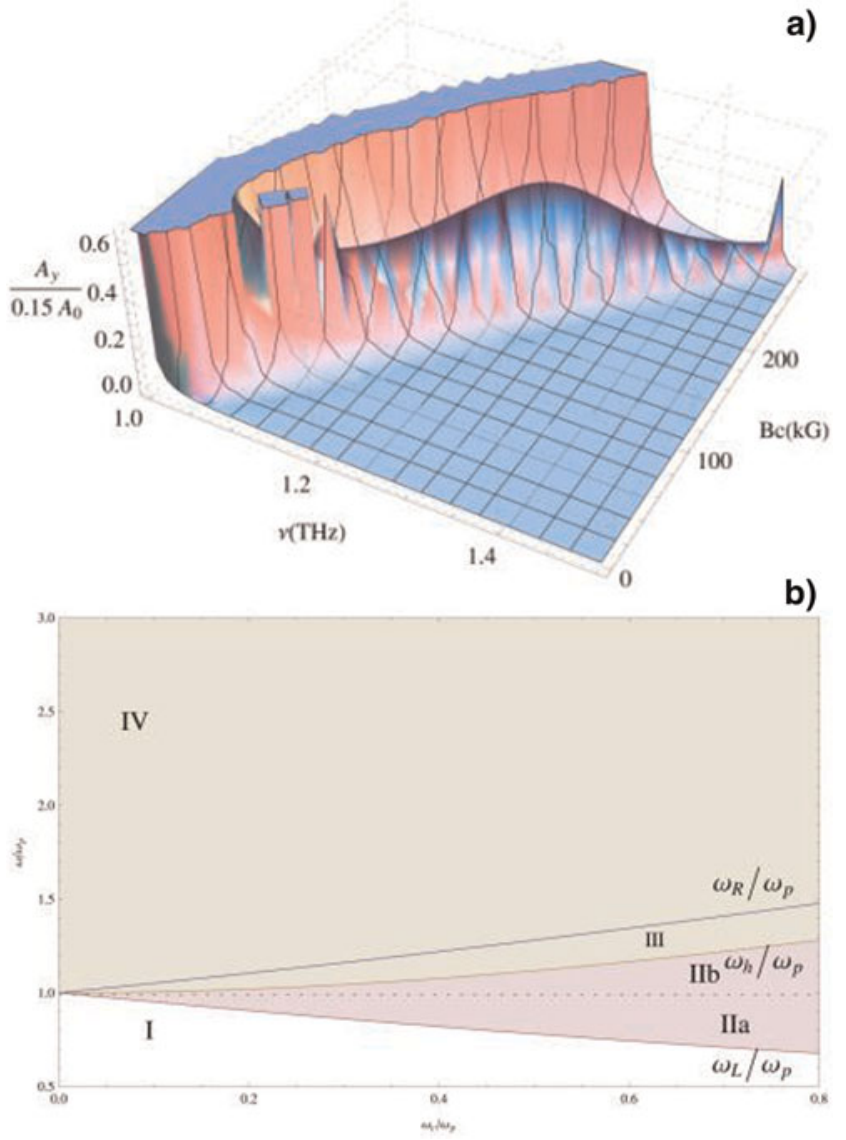

Fig. 3. (a) Sketch of the normalized $\mathrm{THz}$ amplitude $\left(A_{y} / 0.15 A\right)$ as a function of the THz frequency $v(\mathrm{THz})$ and applied static magnetic field $B_{c}$. Other normalized parameters are $a_{0}=5, z=1, y / a_{0}=0.05, v_{1}=0.3, n_{q 0} /$ $n_{0}=0.3$. (b) Sketch of the normalized $\mathrm{THz}$ frequency $\omega / \omega_{p}$ and cyclotron frequency $\omega / \omega_{c}$. by a regime of frequency (Region III of Fig. 3b) in which $\omega^{2} / k^{2}$ becomes negative and propagation of wave stopped.

Peaks of both amplitude mountains are corresponding to resonance conditions which occurs at $\omega \sim \omega_{h}$ and $\omega \sim \omega_{R}$, respectively. The frequency of THZ radiation having maximum amplitude shifts toward higher end of frequency as applied magnetic field increases due to enhanced value $\omega_{h}$ and $\omega_{R}$. Similar type of observation can be drawn from Figure 4 in which normalized amplitude of $\mathrm{THz}$ radiation is plotted as a function of $\mathrm{THz}$ frequency and normalized distance travelled in $z$-direction. THz radiation of frequency $1 \mathrm{THz},(1.1 \mathrm{THz}$ and $1.2 \mathrm{THz}),(1.2 \mathrm{THz}$ and $1.3 \mathrm{THz})$, and $(1.3 \mathrm{THz}$ and $1.5 \mathrm{THz})$ are achieved by applying dc magnetic field $\left(B_{0}\right) 0,107 \mathrm{kG}, 178 \mathrm{kG}$, and $285 \mathrm{kG}$, respectively. Thus maximum energy transfer from beating lasers to $\mathrm{THz}$ radiation takes place at resonance condition and frequency of $\mathrm{THz}$ can be tuned by changing applied magnetic field. As the amplitude of $\mathrm{THz}$ radiation enhances in $z$-direction thus amplitude of excited $\mathrm{THz}$ radiation can also be tuned by varying plasma length.

It can be seen from Figure 5 that $\mathrm{THz}$ amplitude has maximum value on the axis $\left(y / a_{0}=0\right)$ and decreases as one moves off axis which can be attributed to the maximum value of ponderomotive force on the axis. Thus, radiation emitted in the present scheme using $\mathrm{x}$-mode triangular lasers is more colliminated as compared to other schemes (Xie et al., 2006). The amplitude of excited $\mathrm{THz}$ radiation is depending on the ponderomotive force exerting by the laser beams in the plasma (as shown in Eq. (15)). As laser beams propagating in $z$ direction has transverse spatial dependency (triangular shaped) along $y$ direction, the ponderomotive force will also have transverse dependency. Hence this behavior is appearing in our result showing the profile of $\mathrm{THz}$ radiation propagating in $z$ direction and having transverse dependency. It can also be explained in another way: if
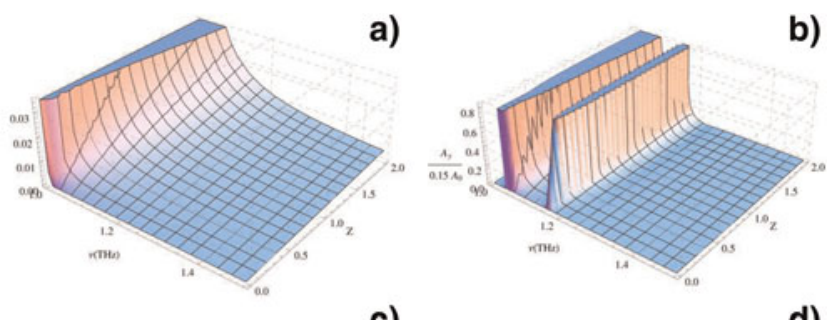

c)

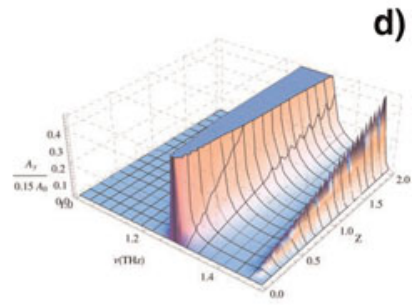

Fig. 4. Plot of the normalized $\mathrm{THz}$ amplitude $\left(A_{y} / 0.15 A_{0}\right)$ as a function of the $\mathrm{THz}$ wave frequency $v(\mathrm{THz})$ and normalized transverse distance $z$ at different cyclotron frequency (a) $\omega_{c}=0.0$, (b) $\omega_{c}=0.3$, (c) $\omega_{c}=0.5$, (d) $\omega_{c}=0.8$. Other normalized parameters are $y / a_{0}=0.05, v_{1}=0.3, n_{q 0} /$ $n_{0}=0.3$ 


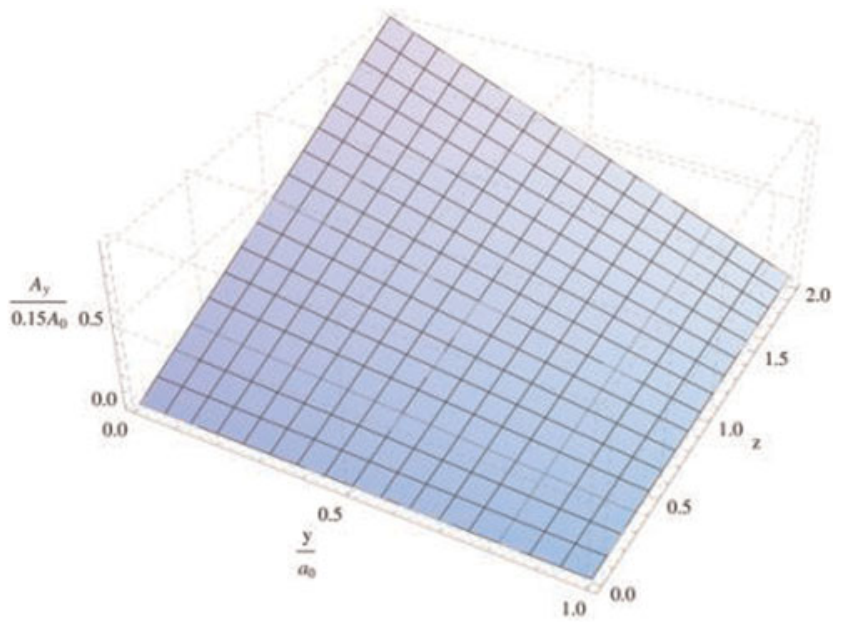

Fig. 5. Plot of the normalized THz amplitude $\left(A_{y} / 0.15 A_{0}\right)$ as a function of the normalized transverse distancezand normalized beam width $y / a_{0}$. Other normalized parameters are $v_{1}=0.3, \omega_{1}=12, n_{q 0} / n_{0}=0.3, \omega_{c}=0.3, a_{0}=5$, $\omega=1.1$.

we choose a particular value of $y$, and solve Eq. (15), we will have the variation of $A_{y}$ as a function of longitudinal direction $z$. Now if we choose another value of $y$, then we will again find different one-dimensional variation of $A_{y}$ because of different value of ponderomotive force (which is dependent on transverse direction $y$ ). Hence due to direct effect of laser profiles and ponderomotive force, excited $\mathrm{THz}$ radiation amplitude $A_{y}$ will also exhibit the transverse dependency.

Finally, we calculated the efficiency of the present scheme based on the energies of the lasers and emitted radiation. The average electromagnetic energy stored in unit volume in electric and magnetic fields (Malik et al. 2012; 2011) yields

$$
\begin{gathered}
W_{p u m p}=\frac{1}{16 \pi} \varepsilon_{0} a_{0} E_{0}^{2}, \\
W_{T H z}=\frac{\varepsilon_{0}}{16 \pi}\left[\frac { 1 } { 4 k } \frac { n _ { q 0 } } { n _ { 0 } } \frac { \omega \omega _ { p } ^ { 2 } } { \omega ^ { 2 } - \omega _ { h } ^ { 2 } } \frac { z } { e c ^ { 2 } } \left[i\left(\frac{\omega^{2}-\omega_{p}^{2}}{\omega}-\left|\frac{\varepsilon_{z y}}{\varepsilon_{z z}}\right| \omega_{c}\right) F_{p y}\right.\right. \\
\left.\left.+\left(\omega_{c}-\omega\left|\frac{\varepsilon_{z y}}{\varepsilon_{z z}}\right|\right) F_{p z}\right]\right]^{2} .
\end{gathered}
$$

Here $W_{\text {pump }}$ is the average energy density of the pump lasers of triangular shape. The ratio of THz energy to pump energy gives the efficiency $\eta$ as below

$$
\begin{gathered}
\eta=\frac{W_{T H z}}{W_{p u m p}}=\left|\frac{A_{y}}{A_{0}}\right|^{2}, \\
\eta=\left[\frac { 1 } { 4 k } \frac { n _ { q 0 } } { n _ { 0 } } \frac { \omega \omega _ { p } ^ { 2 } } { \omega ^ { 2 } - \omega _ { h } ^ { 2 } } \frac { z } { e c ^ { 2 } } \left[i\left(\frac{\omega^{2}-\omega_{p}^{2}}{\omega}-\left|\frac{\varepsilon_{z y}}{\varepsilon_{z z}}\right| \omega_{c}\right) F_{p y}\right.\right. \\
\left.\left.+\left(\omega_{c}-\omega\left|\frac{\varepsilon_{z y}}{\varepsilon_{z z}}\right|\right) F_{p z}\right]\right]^{2} .
\end{gathered}
$$

The efficiency of present scheme is proportional to the square of ripple amplitude, which can be explained on the basis of more charge carriers involved in excitation of nonlinear current responsible for the generation of $\mathrm{THz}$ radiation. The efficiency is also proportional to $z^{2}$ which can be explained as follows: as beating lasers propagate in $z$-direction, they encounter more and more periodic density ripples; higher the number of encountered periodic density ripples better will be the efficacy of three wave coupling required for $\mathrm{THz}$ radiation generation due to efficient momentum transfer (from ripple periodicity to nonlinear current $\vec{J}_{N L}$ to overcome the mismatch in momentum). At the same time, larger number of electrons due to higher number of density ripples will produce larger nonlinear current. The efficiency of $\mathrm{THz}$ radiation generation is plotted as a function of $\mathrm{THz}$ frequency and applied magnetic field in Figure 6. In the present scheme, the efficiency of $\mathrm{THz}$ radiation generation about $2.5 \%$ can be achieved for the frequency range $1-1.3 \mathrm{THz}$ by applying magnetic field about $100 \mathrm{kG}$. It can be noticed that the efficiency of the present scheme is much better than reported by other investigators. For example, Malik et al. (2011) have reported the conversion efficiency about 0.002 by beating of two spatial-Gaussian lasers; Varshney et al. (2013) have reported the conversion efficiency about $10^{-3}$ by beating of two planer $\mathrm{x}$-mode laser; conversion efficiency about 0.006 is achieved by Malik and Malik (2012) by using super Gaussian lasers; whereas present scheme achieved the conversion efficiency about 0.02 by using two triangular shaped x-mode lasers. Hamester et al. (1993; 1994) also obtained the efficiency about $10^{-5}$ with a single laser that is Gaussian in space and time which is two order lesser as compared to the conversion efficiency of present scheme. Wu et al. (2008) reported theoretical as well as numerical simulation of powerful $\mathrm{THz}$ emission using inhomogeneous plasma density. In their model, the maximum energy conversion efficiency at peak intensity $5.48 \times 10^{12}$ $\mathrm{W} / \mathrm{cm}^{2}$ is 0.0005 , which is much lower than present model. Kim et al. (2008) proposed a model for the generation of $\mathrm{THz}$ radiation by irradiating different gases with a symmetry-broken laser field composed of the fundamental and second harmonic laser pulses. In that model, the energy conversion efficiency was about $10^{-5}$, which is three orders lesser as compared to present model.

Effect of electron collision frequency $(v)$ on the efficiency of present scheme as a function of electron temperature is shown in Figure 7. Electron collision frequency depends upon electron temperature by the expression given by $v=$ $\left(z \omega_{p} / 10 N_{D}\right) \ln \left(9 N_{D} / z\right)$, where $N_{D}=4 \pi n_{0} \lambda_{D e}^{3} / 3, \quad \lambda_{D e}$ is the electron Debye length and $n_{0}$ is the electron density in plasmas. As plasma electron temperature increases, electron collision frequency (v) decreases; as a result of which percentage change in efficiency $\left[\left\{\left(\eta-\eta_{v}\right) / \eta\right\} \times 100\right]$ of the present $\mathrm{THz}$ generation scheme decreases. Here, $\eta_{v}$ and $\eta$ are the efficiencies of the scheme with and without electron collisions respectively. All the dimensionless parameters are chosen for $\mathrm{CO}_{2}$ laser $\left(\lambda=1.06 \times 10^{-5} \mathrm{~m}\right)$ having frequency $\omega_{1}=2 \times 10^{14} \mathrm{rad} / \mathrm{s}$ and intensity $I_{L}=$ $2 \times 10^{15} \mathrm{wcm}^{-2}$. 


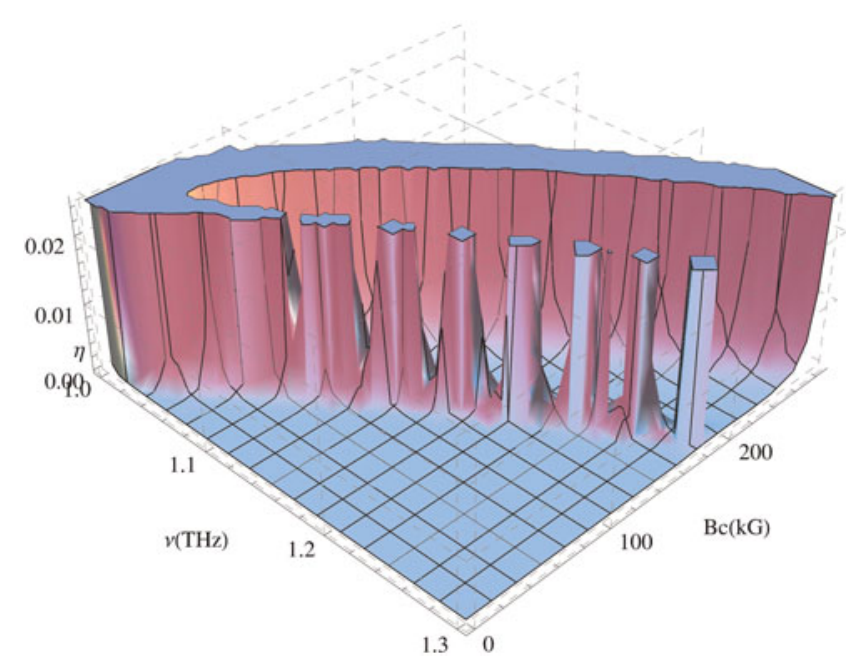

Fig. 6. Variation of efficiency of $\mathrm{THz}$ radiation $\eta$ as a function of the $\mathrm{THz}$ frequency $v(\mathrm{THz})$ and applied static magnetic field $B_{c}$. Other normalized parameters are $v_{1}=0.3, \omega_{1}=12, n_{q 0} / n_{0}=0.3, \omega_{c}=0.3, a_{0}=5, z=1, y / a_{0}=$ 0.5 .

\section{CONCLUSIONS}

Dynamics of $\mathrm{THz}$ radiation generation by beating of two $\mathrm{x}$-mode laser beams in rippled magnetized plasma is studied, when only ponderomotive nonlinearity is operative. Triangular envelop of $\mathrm{x}$-mode laser beams is utilized to enhance beat ponderomotive force acting on plasma electrons. The extra momentum required to excite $\mathrm{THz}$ wave resonantly by beating of two $\mathrm{x}$-mode lasers of frequencies in upper hybrid range is provided by the periodicity of density ripples. The required ripple wave number depends upon $\mathrm{THz}$ frequency and applied magnetic field. It decreases as the $\mathrm{THz}$ frequency increases and increases as the magnetic field increases. Thus, frequency of $\mathrm{THz}$ radiation can be easily tuned by varying plasma density and applied magnetic field. The nonlinear mechanism which generates the $\mathrm{THz}$ radiation can be understood as follows: the nonlinear interaction of laser beams with the plasma having static magnetic field generates the velocity perturbation which leads to density perturbation. Static magnetic field imparts extra longitudinal component to oscillatory velocity of plasma electrons resulting in to transverse ponderomotive force. Both the components of ponderomotive force (Eq. (3) and Eq. (4)) are responsible for density perturbation. As a result, a nonlinear current at beat wave frequency is generated due to coupling between density ripples and velocity components of electron as shown in Eq. (11). Since the difference in laser beam frequencies is in the range of $\mathrm{THz}$ and phase matching conditions are satisfied, the nonlinear current generated at beat frequency generates the desired $\mathrm{THz}$ wave. The $\mathrm{THz}$ amplitude can be controlled by laser plasma parameters and magnitude of applied static magnetic field as shown in Eq. (16). In this scheme, magnetic field plays two roles (1) it controls the phase velocity and group velocity of beating lasers and (2) the polarization of generated $\mathrm{THz}$ wave (Sharma et al., 2010). The $\mathrm{THz}$

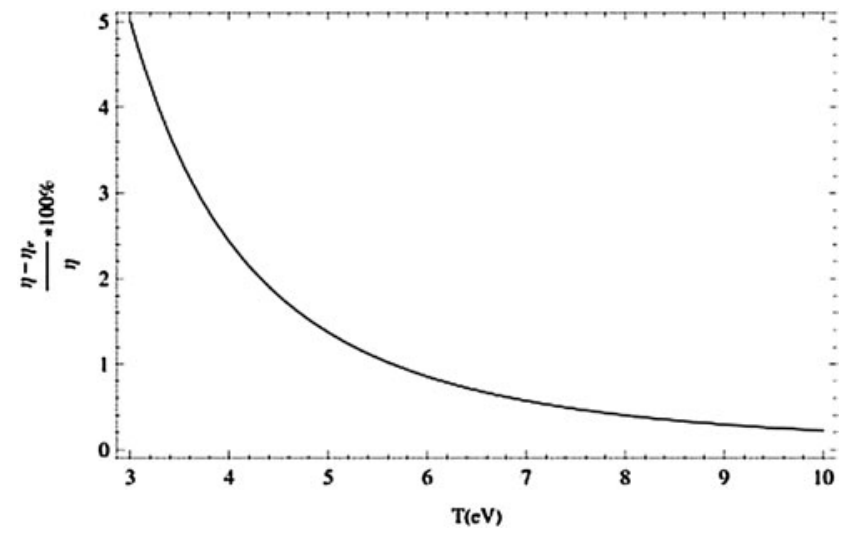

Fig. 7. Percentage change in efficiency of $\mathrm{THz}$ radiation generation as a function of plasma temperature $T(\mathrm{eV})$.

amplitude scales directly to the amplitude of density ripples and maximizes as frequency $(\omega)$ approaches to resonance frequency ( $\approx \omega_{h}$ ). THz radiation emitted by beating of two triangular shaped lasers is more colliminated (Fig. 5) as compared to plane lasers and Gaussian shaped lasers (Malik et al., 2012). In conclusion, we can say that the efficiency, amplitude and tunability of the present $\mathrm{THz}$ generation scheme is better than others.

\section{REFERENCES}

Abo-Bakr, M., Feikes, J., Holldack, K., Kuske, P., Peatman, W.B., Schade, U., Wustefeld, G. \& HüBers, H.W. (2003). Brilliant, coherent far-infrared (THz) synchrotron radiation. Phys. Rev. Lett. 90, 094801.

Bhasin, L. \& TRIPATHI, V.K. (2011). Terahertz generation from laser filaments in the presence of a static electric field in a plasma. Phys. Plasma 18, 123106.

Chen, F.F. (1983). Introduction to Plasma Physics and Controlled Fusion. New York: Plenum Press.

Carr, G.L., Martin, M.C., Mckinney, W.R., Jordan, K., Neil, G.R. \& Williams, G.P. (2002). High-power terahertz radiation from relativistic electrons. Nat. (London) 420, 153.

D'amico, C., Houard, A., Franco, M., Prade, B., Mysyrowicz, A., Couairon, V. \& Tikhonchuk, V.T. (2007). Conical forward THz emission from femtosecond-laser-beam filamentation in air. Phys. Rev. Lett. 98, 235002.

Dragoman, D. \& Dragoman, M. (2004). Time-Frequency signal processing of terahertz pulses. App. Opt. 43, 3848.

Dua, H.W., Chena, M., Shenga, Z.M. \& Zhanga, J. (2011). Numerical studies on terahertz radiation generated from two color laser pulse interaction with gas targets. Laser Part. Beams 29, 447.

Esarey, E., Sprangle, P., Krall, J. \& Ting, A. (1996). Overview of plasma based accelerator concepts. IEEE Trans. Plasma Sci. 24, 252.

GARG, V. \& TRIPATHI, V.K. (2010). Resonant third harmonic generation of an infrared laser in a semiconductor wave guide. Laser Part. Beams 28, 327.

Ghorbanalilu, M. (2012). Second and third harmonics generations in the interaction of strongly magnetized dense plasma with an intense laser beam. Laser Part. Beams 30, 291. 
Giulietti, D., Banfi, G.P., Deha, I., Giulietti, A., Lucchesi, M., Nocera, L. \& Zun, C.Z. (1988). Second harmonic generation in underdense plasma. Laser Part. Beams 6, 141.

Hamster, H., Sullivan, A., Gordon, S., White, W. \& Falcone, R.W. (1993). Subpicosecond, electromagnetic pulses from intense laser-plasma interaction. Phys. Rev. Lett. 71, 2725.

Hamster, H., Sullivan, A., Gordon, S. \& Falcone, R.W. (1994). Short-pulse terahertz radiation from high-intensity-laserproduced plasmas. Phys. Rev. E 49, 671.

Hu, G.Y., Shen, B., Lei, A., LI, R. \& Xu, Z. (2010). Transition Cherenkov radiation of terahertz generated by superluminous ionization front in femtosecond laser filament. Laser Part. Beams 28, 399.

Kim, K.Y., Taylor, A.J., Glownia, T.H. \& Rodriguez, G. (2008). Coherent control of terahertz super continuum generation in ultrafast laser-gas interactions. Nat. Photon. 2, 605.

Kumar, K.K.M. \& Tripathi, V.K. (2013). Third harmonic generation of a nonlinear laser Eigen mode of a self sustained plasma channel. Laser Part. Beams 31, 163.

Leemans, W.P., Geddes, C.G.R., Faure, J., Tóth, C.S. Tilborg, J.V., Schroeder, C.B., Esarey, E., Fubiani, G., Auerbach, D., Marcelis, B., Carnahan, M.A., Kaindl, R.A., Byrd, J. \& MARTIN, M.C. (2003). Observation of Terahertz emission from a laser-plasma accelerated electron bunch crossing a plasmavacuum boundary. Phys. Rev. Lett. 91, 074802.

Liv, C.S. \& TRIPATHI, V.K. (2009). Tunable terahertz radiation from a tunnel ionized magnetized plasma cylinder. J. Appl. Phy. 105, 013313.

Malik, A.K., Malik, H.K. \& Stroth, U. (2012). Terahertz radiation generation by beating of two spatial-Gaussian lasers in the presence of a static magnetic field. Phy. Rev. E 85, 016401.

MaliK, A.K. \& MaliK, H.K. (2012). Strong and collimated THz radiation by super Gaussian lasers. Europ. Phys. Lett. 100, 45001.

MaliK, A.K., MaLIK, H.K. \& NishidA, Y. (2011). Terahertz radiation generation by beating of two spatial-Gaussian lasers. Phys. Lett. A 375, 1191.

Nafil, R.Q., Singh, M., Al-Janabi, A.H. \& Sharma, R. P. (2013). $\mathrm{THz}$ generation by the beating of two high intense laser beams. J. Plasma Phys. 79, 657.

Panwar, A., Ryu, C.M. \& Kumar, A. (2013). Effect of plasma channel non-uniformity on resonant third harmonic generation. Laser Part. Beams 31, 531.

Penano, J., Sprangle, P., Hafizi, B., Gordo, D. \& Serafim, P. (2010). Terahertz generation in plasmas using two-color laser pulses. Phys. Rev. E 81, 026407.
Schillinger, H. \& Sauerbrey, R. (1999). Electrical conductivity of long plasma channels in air generated by self-guided femtosecond laser pulses. Appl. Phys. B 68, 753.

Schroeder, C.B., Esarey, E., Tilborg, J.V. \& Leemans, W.P. (2004). Theory of coherent transition radiation generated at a plasma-vacuum interface. Phys. Rev. E 69, 016501.

Sharma, R.P., Monika, M., Sharma, P., Chauhan, P. \& Jia, A. (2010). Interaction of high power laser beam with magnetized plasma and THz generation. Laser Part. Beams 28, 531.

Sheng, Z.M., Wu, H. C., Li, K. \& Zhang, J. (2004). Terahertz radiation from the vacuum-plasma interface driven by ultrashort intense laser pulses. Phys. Rev. E 69, 025401.

Sheng, Z.M., Mima, K. \& Zhang, J. (2005). Powerful terahertz emission from laser wake fields excited in inhomogeneous plasmas. Phys. Plasmas 12, 123103.

SiEgEL, P.H. (2002). Terahertz technology. IEEE Trans. Microwave Theo. Tech. 50, 910.

Sizov, F. (2010). THz radiation sensors. Opt. Electron. Rev. 18, 10.

Sprangle, P., Penano, J. R., Hafizi, B. \& Kapetanakos, C.A. (2004). Ultrashort laser pulses and electromagnetic pulse generation in air and on dielectric surfaces. Phys. Rev. E 69, 066415.

Tani, M., Gu, P., Hyodo, M., Sakai, K. \& Hidaka, T. (2000). Generation of coherent terahertz radiation by photo mixing of dualmode lasers. Opt. Quan. Electron. 32, 503-520.

Tripathi, V.K. \& LiU, C.S. (1990). Plasma effects in a free electron laser. IEEE Trans. Plasma Sci. 18, 466.

Verma, U. \& Sharma, A.K. (2009). Laser second harmonic generation in a rippled density plasma in the presence of azimuthal magnetic field. Laser Part. Beams 27, 719.

Verma, U. \& Sharma, A.K. (2011). Nonlinear electromagnetic Eigen modes of a self created magnetized plasma channel and its stimulated Raman scattering. Laser Part. Beams 29, 471.

Varshney, P., Sajal, V., Singh, K.P., Kumar, R. \& Sharma, N.K. (2013). Strong terahertz radiation generation by beating of extra-ordinary mode lasers in a rippled density magnetized plasma. Laser Part. Beams 31, 337.

Varshney, P., Sajal, V., Chauhan, P., Kumar, R. \& Sharma, N.K. (2014). Effects of transverse static electric field on terahertz radiation generation by beating of two transversely modulated Gaussian laser beams in a plasma. Laser Part. Beams 32, 375.

Wu, H.C., Sheng, Z.M. \& Zhang, J. (2008). Single-cycle powerful megawatt to gigawatt terahertz pulse radiated from a wavelength-scale plasma oscillator. Phys. Rev. E 77, 046405.

XIE, X., DAI, J.M. \& Zhang, X.C. (2006). Coherent control of THz wave generation in ambient air. Phys. Rev. Lett. 96, 075005. 